Furthermore, it has been shown that in asthmatics treated with budesonide in a dose-escalation study, AMP was more sensitive than methacholine in detecting differences in AHR by approximately one doubling dilution [5].

Despite improvements in airway hyperresponsiveness, clarithromycin conferred no worthwhile improvement upon lung function. Therefore, the study by KosTADIMA et al. [1] provides a timely reminder that monitoring the effects of asthma pharmacotherapy based solely on lung function can miss potentially beneficial effects upon airway hyperresponsiveness and underlying inflammation [6]. Further long-term studies are needed to assess whether effects upon airway hyperresponsiveness with clarithromycin translate into clinically meaningful reductions in exacerbations. Moreover, whether macrolides confer benefit upon inflammatory biomarkers, such as airway hyperresponsiveness to adenosine monophosphate and sputum eosinophilia, requires investigation.

G.P. Currie*, D.K.C. Lee ${ }^{\#}$

*Dept of Respiratory Medicine, Aberdeen Royal Infirmary, Aberdeen, and ${ }^{\#}$ Dept of Respiratory Medicine, Ipswich Hospital, Ipswich, UK.

\section{References}

1. Kostadima E, Tsiodras S, Alexopoulos EI, et al. Clarithromycin reduces the severity of bronchial hyperresponsiveness in patients with asthma. Eur Respir $J$ 2004; 23: 714-717.

2. Cockcroft DW. How best to measure airway responsiveness. Am J Respir Crit Care Med 2001; 163: 1514-1515.

3. Van Den Berge M, Meijer RJ, Kerstjens HA, et al. PC(20) adenosine $5^{\prime}$-monophosphate is more closely associated with airway inflammation in asthma than PC(20) methacholine. Am J Respir Crit Care Med 2001; 163: 1546-1550.

4. De Meer G, Heederik D, Postma DS. Bronchial responsiveness to adenosine 5'-monophosphate (AMP) and methacholine differ in their relationship with airway allergy and baseline FEV(1). Am J Respir Crit Care Med 2002; 165: 327331.

5. Wilson AM, Lipworth BJ. Dose-response evaluation of the therapeutic index for inhaled budesonide in patients with mild-to-moderate asthma. Am J Med 2000; 108: 269275.

6. Currie GP, Lee DKC, Haggart K, Bates CE, Lipworth BJ. Effects of montelukast on surrogate inflammatory markers in corticosteroid treated patients with asthma. Am J Respir Crit Care Med 2003; 167: 1232-1238.
From the authors:

In our study [1], an 8-week treatment with clarithromycin was associated with a significant improvement in airway hyperresponsiveness to methacholine in asthmatic patients. As G.P. Currie and D.K.C. Lee correctly point out, this significant change was not accompanied by a clinically important increase in indices of expiratory flow function. We agree that further long-term clinical trials are necessary to identify any beneficial effects of clarithromycin on symptoms of asthma and on markers of airway inflammation.

We chose inhalation of methacholine, a direct stimulus, to detect changes in airway hyperresponsiveness in asthmatics treated with clarithromycin. Provocation with AMP (indirect bronchial challenge) could have also been used. Provocative concentration causing a $20 \%$ fall in forced expiratory volume in one second AMP reflects the extent of airway inflammation due to asthma more closely than provocative concentration causing a $20 \%$ fall in forced expiratory volume in one second methacholine [2]. In addition, as G.P. Currie and D.K.C. Lee appropriately emphasise in their letter, provocation with AMP is more sensitive than provocation with methacholine in detecting changes in airway hyperresponsiveness following anti-inflammatory treatment [3]. The fact that we were able to detect improvement in airway hyperresponsiveness using the less sensitive direct bronchial challenge further supports a potentially important role of clarithromycin in the treatment of asthmatic airway disease.

E. Kostadima*, S. Tsiodras" ${ }^{\#}$, E. Alexopoulos ${ }^{\top}$, A. Kaditis ${ }^{\top}$, I. Mavrou $^{+}$, N. Georgatou ${ }^{+}$, A. Papamichalopoulos ${ }^{+}$

*Dept of Critical Care Medicine and Dept of Pediatrics, University of Thassaly School of Medicine and Larissa University Hospital, ${ }^{\text {4th }}$ Academic Dept of Internal Medicine, University of Athens School of Medicine, and ${ }^{+} 10$ th Respiratory Dept, Athens Chest Hospital, Athens, Greece.

\section{References}

1. Kostadima E, Tsiodras S, Alexopoulos E, et al. Clarithromycin reduces the severity of bronchial hyperresponsiveness in patients with asthma. Eur Respir $J$ 2004; 23: 714-717.

2. Van Den Berge M, Meijer RJ, et al. PC(20) adenosine 5monophosphate is more closely associated with airway inflammation in asthma than $\mathrm{PC}(20)$ methacholine. $\mathrm{Am}$ $J$ Respir Crit Care Med 2001; 163: 1546-1550.

3. Wilson AM, Lipworth BJ. Dose-response evaluation of the therapeutic index for inhaled budesonide in patients with mild-to-moderate asthma. Am J Med 2000; 108: 269-275.

\title{
"Chronic obstructive pulmonary disease": the diagnostic last refuge of the intellectually challenged?
}

\section{To the Editor:}

SuISSA [1] makes an important contribution to the debate on inhaled steroids and chronic obstructive pulmonary disease (COPD) in identifying, in an observational study, an artificial increase in death rate of the reference group leading to a spurious appearance of effectiveness. SUISSA [1] refers, in discussion, to what I believe to be an equally important and curiously neglected source of bias, the unintended inclusion of asthma patients when selecting patients for studies from administrative databases using only age and bronchodilator use to define the disease entity. The term COPD is a tooconvenient shorthand label for a group of conditions that can be shown, even with standard investigative tools, to be of 
extreme heterogeneity and often includes patients with coexisting pathologies, one obvious example being asthma and emphysema. The assumption that it defines anything resembling a cohesive entity in terms of aetiology or pathogenesis is anti-intellectual, and the obvious bias in studies of this nature is just one example. The demonstration that inhaled corticosteroids are effective in "COPD" defined in this way tells us nothing about their efficacy (or more likely lack of it) in smoking-related airflow limitation. "COPD" should be the acronym for "Cop-out On Proper Diagnosis". Not only does it confound the proper assessment of common disease processes, it prevents the effective evaluation of rarer ones, and probably the recognition of new and important entities; no wonder $\alpha$-1 antitrypsin-deficient emphysema was discovered by a biochemist, not a clinician.

\section{P. Barber}

Bronchology Unit, Wythenshawe hospital, Manchester, UK.

\section{References}

1. Suissa S. Inhaled steroids and mortality in COPD: bias from unaccounted immortal time. Eur Respir J 2004; 23: 391-395.

DOI: $10.1183 / 09031936.04 .10062504$

\section{From the authors:}

P. Barber correctly points out the difficulties in identifying chronic obstructive pulmonary disease (COPD) patients using administrative databases, and especially in distinguishing between COPD and asthma. This is particularly important when assessing the impact of inhaled corticosteroids because these drugs have been shown in randomised trials to be extremely effective in asthma but not in COPD. Thus, a study based on a mixed population that includes both asthma and COPD patients will result in an average effect for inhaled corticosteroids.

To maximally ensure the accuracy of a first-time COPD diagnosis for cohort entry, we used three criteria: 1) 55 yrs of age or over; 2) three or more prescriptions on at least two different dates for a bronchodilator within a 1-yr period; and 3) no other prescriptions for bronchodilators or other asthma drugs during the 5-yr period prior to the three defining prescriptions $[1,2]$. With these criteria, we were confident that the proportion of asthma patients would be kept to a negligible level.

In our previous research on asthma, we effectively used similar criteria to study asthma, with the difference that age at cohort entry (diagnosis) was restricted to between 5 and 44 yrs, thus reducing the possibility of including COPD patients [3-5].

While observational databases studies are challenging, they are indispensable to complement other types of studies into the effects of drugs. As P. Barber notes, scientific rigor must be introduced in their design and analysis, which can be best achieved by intense collaborations between clinicians and methodologists. We have and will continue to work in this direction.

\section{S. Suissa}

Division of Clinical Epidemiology, McGill University, Royal Victoria Hospital, Montreal, Quebec, Canada.

\section{References}

1. Suissa S, Assimes T, Ernst P. Inhaled short acting beta agonist use in COPD and the risk of acute myocardial infarction. Thorax 2003; 58: 43-46.

2. de Melo MN, Ernst P, Suissa S. Inhaled corticosteroids and the risk of a first exacerbation in COPD patients. Eur Respir $J$ 2004; 23: 692-697.

3. Suissa S, Ernst P, Benayoun B, Baltzan M, Cai B. Low-dose inhaled corticosteroids and the prevention of death from asthma. N Engl J Med 2000; 343: 332-336.

4. Suissa S, Ernst P, Kezouh A. Regular use of inhaled corticosteroids and the long term prevention of hospitalisation for asthma. Thorax 2002; 57: 880-884.

5. Suissa S, Assimes T, Brassard P, Ernst P. Inhaled corticosteroid use in asthma and the prevention of myocardial infarction. Am J Med 2003; 115: 377-381.

\section{Protective effect on AMP airway responsiveness after a single dose of fluticasone}

\section{To the Editor:}

We read with great interest the article by LuIJK et al. [1] describing the time-course of substantial protective effects after a single dose of inhaled fluticasone propionate (FP) on adenosine-5'-monophosphate (AMP)-induced bronchoconstriction in asthma. No change was observed in terms of exhaled nitric oxide (eNO) levels.

These findings would further support the current opinion that airway responsiveness to AMP is more sensitive than eNO, together with other noninvasive markers of airway inflammation, in assessing the response to anti-inflammatory treatments [2, 3]. To this end, it must be emphasised that inhaled glucocorticosteroids (GCS) have been shown to increase the provocative concentration causing a $20 \%$ fall in forced expiratory volume in one second (PC20) threshold of AMP in a dose-dependent manner, whereas the dose response for eNO, together with many other inflammatory markers, and measures of lung function have all been shown to exhibit a plateau effect at lower doses [4]. Thus, the changes observed with eNO are not truly dose dependent, whereas the degree of anti-inflammatory effects of AMP challenge are directly proportional to the dose of inhaled GCS. The idea that AMP bronchial provocation seems to offer substantial advantages over eNO has been recently supported by the work of PRIETO et al. [5], in which airway responsiveness to inhaled AMP is a useful predictor for safe dose reduction of inhaled GCS in patients with asthma. 